\title{
Noisy Iris Recognition Based on Deep Neural Network
}

\author{
Eman M. Omran \\ A National Center for Radiation \\ Research and Technology \\ (NCRRT), Egyptian Atomic Energy \\ Authority (EAEA), Cairo 11787, \\ Egypt.
}

\author{
Sameh A. Napoleon \\ Mathematics and Computer \\ Science Department, Faculty of \\ Science, Menoufia University, \\ Shebin El-Koom, 32511, Egypt
}

\author{
Nabil A. Ismail \\ Computer Science \& \\ Engineering Dept., Menoufia \\ University, Egypt.
}

\author{
Randa F. Soliman \\ Mathematics and Computer \\ Science Department, Faculty of \\ Science, Menoufia University, \\ Shebin El-Koom, 32511, Egypt
}

\author{
El-Sayed M. El-Rabaie \\ Electronics and Electrical \\ Communications Engineering \\ Department, Faculty of Electronic \\ Engineering, Menouf 32951, \\ Menoufia University.
}

\author{
Ayman A. Eisa \\ A National Center for Radiation \\ Research and Technology \\ (NCRRT), Egyptian Atomic Energy \\ Authority (EAEA), Cairo 11787, \\ Egypt.
}

\author{
Maryam Mostafa Salah \\ Mathematics and Computer \\ Science Department, Faculty of \\ Science, Menoufia University, \\ Shebin El-Koom, 32511, Egypt
}

\author{
Mustafa M. AbdeEInaby \\ Department of Electronics and \\ Communications Engineering, \\ Tanta University, Egypt
}

\author{
Fathi abd El-samie \\ Department of Electronics and \\ Electrical Communications \\ Engineering, Faculty of Electronic \\ Engineering, Menoufia University, \\ Menouf 32952, Egypt
}

\begin{abstract}
Iris recognition is one of the Biometric systems used for persons identification based on their special iris traits, which are unique featuresfor each individual. It is clear that the progress in deep learning show how efficient the extracted features from convolutional neural networks (CNNs) to describe the complex image patterns. However, the influence of noise is a serious problem in most image processing systems. It may ariseto the iris recognition systems due to environmental conditions that can affects the features extracted from the iris images. Hence, the objective of this paper is to study the performance of CNNs based Deep learning (Alex net, Vgg16 and Vgg19) when used for iris recognition with the presence of noise and compares it with Masek algorithm. Simulation results reveal that using the deep learning greatly improves iris recognition accuracy for Alex CNN. We achieve $100 \%$, $100 \%, 88.9 \%$ for interval, lamp and twins datasets respectively.
\end{abstract}

\section{Introduction}

Biometric recognition systems are based on the behavioral and physiological characteristics of the person. These characteristics are specialtraits assigned to each individual like Ear, Fingerprint, Face, Gait,Voice, Palm print andDNA. In the $19^{\text {th }}$ centurythe fingerprint utilizedin the criminal cases in Paris then the other types of biometric traits like face and iris have been prayed an attention [1], [2].Iris is the circular part that located between pupil and sclera,foriris recognition the iris is firstly localized, normalized to a fixed rectangular block, the features are extracted and matching. The most significant step is the iris localization, because all other steps depend on its accuracy [3].
Several techniques are developed for iris localization for instance Integro-differential operator, Circular Hough Transform (CHT), Distance Regularized Level Set Evolution (DRLSE).Daugman [4] proposed an iris localization technique based on "Integro-differential" operator. Later numbers of researches were done to enhance this method.Thosemodifications arerepresented in [3]. Wildes [5] developed the (CHT) that has been used by Masek [6]. The Active Contour (AC)is another iris localization algorithm, specifically (DRLSE) [7].

Among these entire techniques, CHT algorithm that explained in [8], [9] achieves higher recognition accuracies with allCASIA-Iris V3 images, and it can robust in the presence of blurring and noise in image. CHT works in the parameter space because we can locate the edge points of iris in parameter space [8]. On the other hand, there are two problems in CHT, first, it requires threshold values for edge detection and it has a heavy computational cost.

Recently there are a few trails to appropriate the fundamentals of CNNs based deep learning to the iris recognition task [10], [11]. Simply to design a CNN architecture for iris recognition task without inherent visions would be very difficult for several reasons firstly, largescale iris datasets not available in the public domain secondly, how optimal to choose the ad hoc options for CNN model like number of layers, arrangement of layers , training option and so on. Therefore, in this paper instead of designing and training new model of $\mathrm{CNN}$ for iris recognition task we using pre- trained CNNs. Researchers have confirmed that these pre-trained $\mathrm{CNN}$ features are more effective in the field of computer vision tasks, like face recognition and classification, visual instance retrieval and action recognition [12]. In this paper, we will examine the performance of those pre-trained $\mathrm{CNNs}$ for iris 
recognition with the presence of noise to ensure the stability of these networks.

The fundamental contributions of this paper are:

- The proposed approach archives high superior recognition accuracy for iris recognition (IR).

- It exploits pre-trained $\mathrm{CNN}$ to provide stable IR performance with the presence of noise.

This paper is organized as follows: in Section 2 we talking about related work for CNNs in literature for IR and the pre-trained CNNs that we have used in this work. Section 3 presents our proposed framework and the experimental results. Section 4 concludes the paper.

\section{Related Work}

CNNs are convolutional neural networks based deep learning; they are extremely effective in image recognition and classification, and object detection and recognition [13], [14]. Convolutional neural network $(\mathrm{CNN})$ is one of deep learning methods, which designed for purpose of image and video processing. CNN is formed by staking multi layers, which contain repetitive blocks of neurons and applied in hierarchical manner across images, CNNs can automatically learn image feature and they have exceeded many traditional feature techniques [15].

CNNs became popular whenKrizhesky et al. [16] proposed a CNN called AlexNet. AlexNet is a version of the LeNet with a deeper configuration;however it is trained on a large dataset contains 14 million imageswith graphical processing unit (GPUs). On the other hand, manyefficient and novellearning techniques have been proposed to make CNNsmore powerful and deeper [17],[18], [19], [20].A number of deep networks have been suggested for enhancing the iris recognition accuracy. Liu et al. [21] suggested a Deep neural network consists of 9 layers one pairwise filter layer, one convolutional layer,two normalization layers, two pooling layers, two local layers and one fully-connected layer. This network attained a very hopeful recognition accuracy on CASIA [22] datasets.

Gangwar et al. [10] proposedtwo more developed networks for iris recognition. The first deep network, enclosedeight convolutional layers (batch normalization layer followed each one), 4 pooling layers, 3 fully connected layers and two drop-out layers. In the second deep network, they added two beginning layers to increase the capability of model. These two deep networks revealedbetterrecognition performance on NDCrossSensor-Iris-2013and ND-IRIS-0405 [23] datasets.

Recently, CNNs have also been utilized for gender identification [24], iris segmentation [25], [26] and spoofperception [27], [28].
As well as, CNNs such DeepIrisNet[29] and DeepIrisNet [20] alsorevealed promising recognition results, but with major limit which is the choice on the number of designed layers is restricted by the training samplesnumber. However, Stacking varies layers to form a new $\mathrm{CNN}$ for providing iris recognition would be difficult due to the lack of large-scale iris datasets in the public domain and how to choose the appropriate layer design to give better performance. Therefore,transfer learning approach can be used.In fact, the pre-trained CNN that trained on ImageNet dataset [30] can be successfully transferred to perform several computer vision tasks [10]. Minaee et al. [30] presented that the VGGnetwork, that utilized to classify objectscan work efficiently for iris recognition.

\section{CNNs in Use}

The extracted Features from CNNs utilize very well for recognition and classification tasks. First, we review some CNN models and then explain our framework for noisy iris recognition based on these CNN Features.

Alex Net: Alex Net is one version of CNN[15], which contains 5 convolutional layers and has a better performance whenused for training large-scale dataset and it can be trained with GPUs which can decrease the training time ten times. Better performance can be achieved by modifying Alex Net hyperparametersthrough the transfer learning.

VGG Net: Another version of $\mathrm{CNN}$ which is characterized with good performance and simplicity [17]. The two most popular versions of VGG net are VGG-16 and VGG-19 that contain 16 and 19 convolutional layers, respectively.

\section{Experimental Results}

\subsection{CASIA-Iris Recognition Framework}

In this paper, we study the effect of noise on the recognition accuracy, which give indicator to the effectiveness, and robustness of the CNNs used Fig. 1 show the framework we employed to examine the performance of the pre-trained CNNs with the presence of noise. Firstly, we enhance our pictures resolution by passing them throw Adaptive histogram Equalization Filter before feeding them to the CNN used. Secondly, fine tuning these pre-trained $\mathrm{CNNs}$ to learn the features on a new collection of iris images, which sustain high recognition accuracy. Finally, we were applying (AWGN)with variance value from 0.01 to 0.07 on the images before entered to the net to measure the effectiveness of these CNNs to classify noisy iris successfully. In Fig. 1, AHE refers to Adaptive Histogram Equalization while AWGN is the Additive White Gaussian Noise.

Fine-Tuning: Due to a small amount of training images, build up and training a new $\mathrm{CNN}$ network may be time consuming. Instead, we fine-tune an existing pre-trained CNNs network for training iris image. This technique is called transfer learning. Here we take all the layers from the pre-trained $\mathrm{CNN}$ network except the last 3 then we add a new fully connected layer suitable to the number of classes in the new database, a softmax layer, and a classification output layer. 
SVM Classification:Simple multi-class Support Vector Machine (SVM) [31] is used for classification process due to its efficiency in image classification.

\subsection{Database}

We conducted our experiments on CASIA-Iris V3 database which contains 22034 iris images [22].It contains interval, lamp, and twins databases as shown in Fig. 2, the resolution of interval is $320 \times 280$,twins and lamp has a resolution of $640 \times 480$.

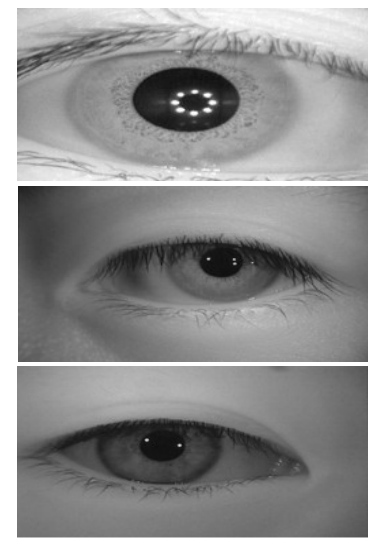

Figure 2 Samples of the three types of CASIA-Iris V3

\subsection{Experimental Setup}

We select $70 \%$ of the iris images for training and $(30 \%)$ for testing. It must be noted that the iris features used to train a multi-class SVMs; and we start training with 'InitialLearnRate' of about 0.0001, Stochastic Gradient Descent (SGD) used for weight update, the 'MiniBatchSize' size is 32 and epochs 20. We implemented our experiments using MATLAB R2017a on a platform with the following specifications shown in Table 1.

Table 1 Platform specifications.

\begin{tabular}{cc}
\hline System & 64-bit Win 10 \\
Processor & Intel Xeon 5670, 12 \\
& cores \\
Accelerator & NVIDIA GeForce GTX \\
& 1070 \\
Installed & $32 \mathrm{~GB}$ \\
Memory & \\
\hline
\end{tabular}

\subsection{Experimental Results}

We used three models of CNNs based deep learning nets, which are Alex net, Vgg16 and Vgg19. The evaluation of the performance to these nets is done by calculate the recognition accuracy then compared it with Masek result. Table 2 Illustrates the recognition accuracy of CNNs used with compared to Masek result.
To improve the recognition accuracy we pass the iris images through adaptive histogram equalization to enhance the images resolution and fed them to the fine-tuned pre-trained $\mathrm{CNN}$ to learn the features on a new collection of iris images. The recognition accuracy after tuning is shown in Table. 3 .

After that, we studied the effect of noise on the recognition accuracy of the models by applying Additive White Gaussian Noise (AWGN) with variance value from 0.01 to 0.07 on the enhanced images before feeding them to the net. Finally, the result of studying the effect of noise on the recognition accuracy of Alex net, VGG16, 19 nets and Masek are given in Tables 4, 5, 6 and 7.

\section{Performance Analysis}

To evaluate the performance of each model, the recognition accuracy estimated after a feature vector, which represents the iris, generated from the output of each layer. The recognition accuracies before and after tuning are illustrated in table 1 and table 2.Among all three CNNs based deep learning, Alex net achieves the highest recognition accuracies of $83.79 \%, 99.5 \%$ and $61.19 \%$ for CASIA-Iris V3 interval, lamp, and twins datasets respectively. Appling Adaptive histogram Equalization on the images before entering to the $\mathrm{CNN}$ model then fine-tuning the net can be improved the recognition accuracy efficiently with compared to Masek algorithm, which has a maximum recognition accuracy of $81.81 \%$ for CASIA-Iris V3 datasets interval. These modifications can improve the recognition accuracies to $100 \%, 100 \%$ and $88.9 \%$ for Alex net on CASIA-IrisV3 interval, lamp and twins datasets respectively .Vgg 16 achieves recognition accuracies of $97.88 \%, 100 \%$ and $94.5 \%$ respectively to interval, lamp and twins datasets. Also recognition accuracies of $\operatorname{Vgg} 19$ improved to achieve 97.5\%, $100 \%$ and $85.6 \%$ for interval, lamp and twins datasets respectively. To ensure the effectiveness and robustness of the CNNs models used, we are applying Additive White Gaussian Noise (AWGN) with variance value from 0.01 to 0.07 on the enhanced images before entering them to the net and compare with Masek result. Figure 4(a,b,c and d) ensures the stability of recognition accuracies of CNNs used in the presence of noise than the traditional method of Masek algorithm and this confirm that CNN proved to be awarded in the field of iris recognition. This is because of increasing the noise variance, the recognition accuracies related to CNNs are slightly affected but in Masek algorithm, the recognition accuracies go towered zero. 


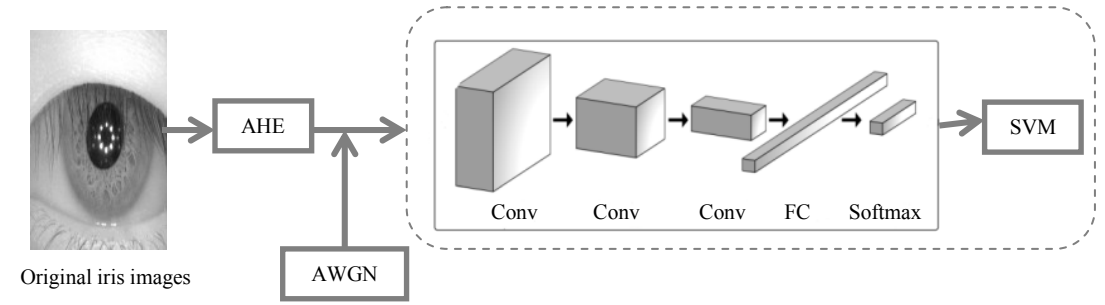

Fig. 1 CASIA-Iris recognition frame work with the presence of noise

Table 2 Recognition accuracy results of CNNs without tunning.

\begin{tabular}{cccc}
\hline Model /database & Interval & Lamp & Twins \\
\hline Alex net & $83.79 \%$ & $99.5 \%$ & $61.19 \%$ \\
Vgg16 & $89 \%$ & $99.5 \%$ & $66.9 \%$ \\
Vgg19 & $84.73 \%$ & $98.64 \%$ & $65.6 \%$ \\
Masek & $81.81 \%$ & $55.8 \%$ & $72.72 \%$ \\
\hline Table 3 Recognition accuracy results of CNNs with fine-tuning. \\
\hline Model /database & Interval & Lamp & Twins \\
\hline Alex net & $100 \%$ & $100 \%$ & $88.9 \%$ \\
Vgg16 & $97.88 \%$ & $100 \%$ & $94.5 \%$ \\
Vgg19 & $97.5 \%$ & $100 \%$ & $85.6 \%$ \\
Masek & $81.81 \%$ & $55.8 \%$ & $72.72 \%$ \\
\hline Table 4 Alex net model performance in the presence of noise reduction \\
\hline 0.01 & Interval & Lamp & Twins \\
\hline 0.02 & $95.37 \%$ & $99.87 \%$ & $82.94 \%$ \\
$\mathbf{0 . 0 3}$ & $92.54 \%$ & $100 \%$ & $97.84 \%$ \\
$\mathbf{0 . 0 4}$ & $91.83 \%$ & $99.87 \%$ & $97.18 \%$ \\
$\mathbf{0 . 0 5}$ & $94.32 \%$ & $99.87 \%$ & $81.32 \%$ \\
$\mathbf{0 . 0 6}$ & $94.85 \%$ & $99.60 \%$ & $81.32 \%$ \\
$\mathbf{0 . 0 7}$ & $92.18 \%$ & $99.60 \%$ & $80.15 \%$ \\
\hline Tariance/database & $89.88 \%$ & $99.60 \%$ & $78.68 \%$ \\
\hline
\end{tabular}

Table 5 Vgg16 model performance in the presence of noise reduction

\begin{tabular}{cccc}
\hline Variance/database & Interval & Lamp & Twins \\
\hline $\mathbf{0 . 0 1}$ & $95.01 \%$ & $100 \%$ & $92.35 \%$ \\
$\mathbf{0 . 0 2}$ & $94.49 \%$ & $100 \%$ & $91.54 \%$ \\
$\mathbf{0 . 0 3}$ & $93.25 \%$ & $100 \%$ & $89.96 \%$ \\
$\mathbf{0 . 0 4}$ & $92.54 \%$ & $100 \%$ & $89.17 \%$ \\
$\mathbf{0 . 0 5}$ & $92.18 \%$ & $100 \%$ & $88.64 \%$ \\
$\mathbf{0 . 0 6}$ & $91.95 \%$ & $100 \%$ & $88.34 \%$ \\
$\mathbf{0 . 0 7}$ & $91.54 \%$ & $100 \%$ & $87.97 \%$ \\
\hline
\end{tabular}

Table $6 \mathrm{Vgg} 19$ model performance in the presence of noise reduction

\begin{tabular}{cccc}
\hline Variance/database & Interval & Lamp & Twins \\
\hline $\mathbf{0 . 0 1}$ & $95.01 \%$ & $100 \%$ & $83.56 \%$ \\
$\mathbf{0 . 0 2}$ & $94.73 \%$ & $100 \%$ & $82.40 \%$ \\
$\mathbf{0 . 0 3}$ & $94.32 \%$ & $100 \%$ & $82.25 \%$ \\
$\mathbf{0 . 0 4}$ & $93.98 \%$ & $100 \%$ & $81.98 \%$ \\
$\mathbf{0 . 0 5}$ & $93.61 \%$ & $100 \%$ & $81.93 \%$ \\
$\mathbf{0 . 0 6}$ & $93.61 \%$ & $100 \%$ & $81.41 \%$ \\
$\mathbf{0 . 0 7}$ & $93.61 \%$ & $100 \%$ & $81.41 \%$ \\
\hline
\end{tabular}

Table 7 Masek Algorithm performance in the presence of noise reduction

\begin{tabular}{cccc}
\hline Variance/database & Interval & Lamp & Twins \\
\hline $\mathbf{0 . 0 1}$ & $77.27 \%$ & $3 \%$ & $37.5 \%$ \\
$\mathbf{0 . 0 2}$ & $54.54 \%$ & $0 \%$ & $4.1 \%$ \\
$\mathbf{0 . 0 3}$ & $22.72 \%$ & $0 \%$ & $0 \%$ \\
$\mathbf{0 . 0 4}$ & $18.18 \%$ & $0 \%$ & $0 \%$ \\
$\mathbf{0 . 0 5}$ & $13.63 \%$ & $0 \%$ & $0 \%$ \\
$\mathbf{0 . 0 6}$ & $9 \%$ & $0 \%$ & $0 \%$ \\
$\mathbf{0 . 0 7}$ & $4.5 \%$ & $0 \%$ & $0 \%$ \\
\hline
\end{tabular}




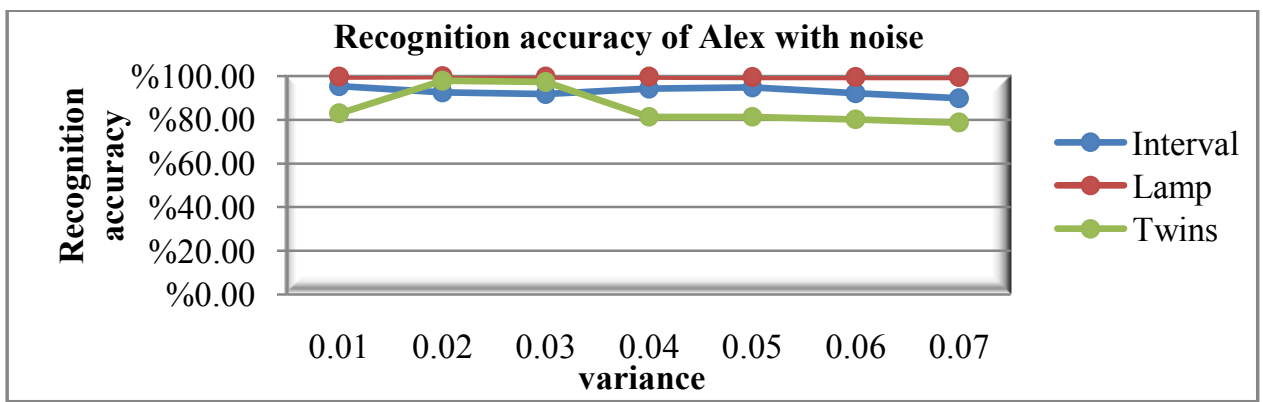

(a)

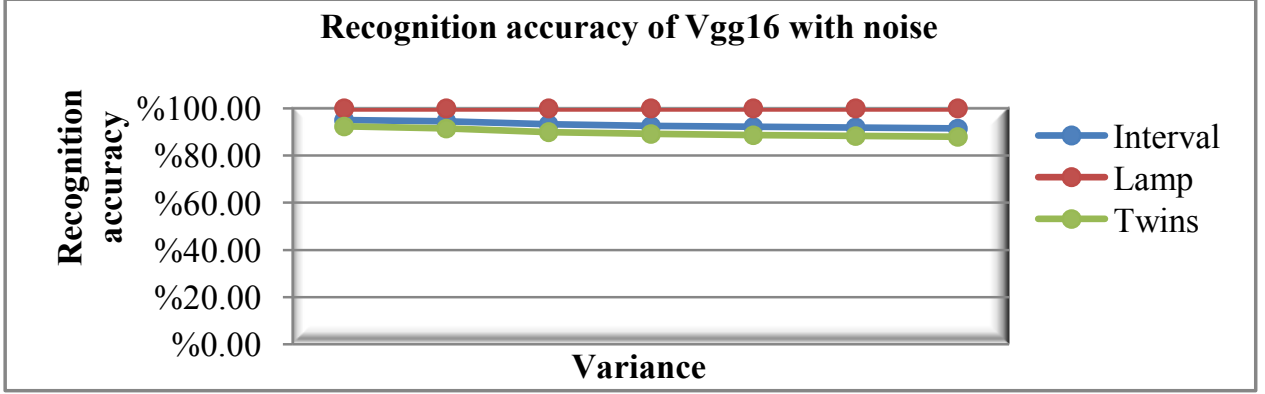

(b)

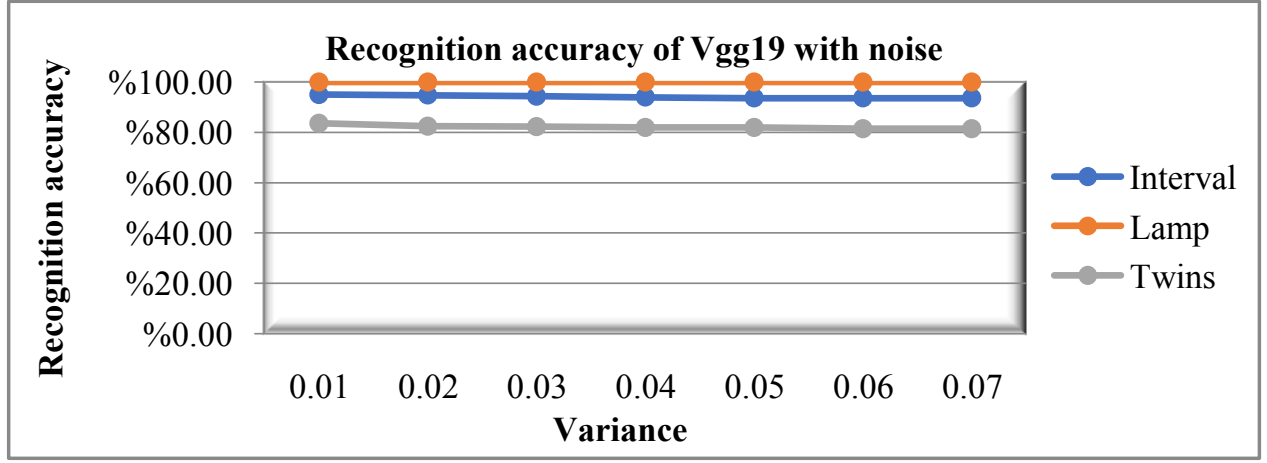

(c)

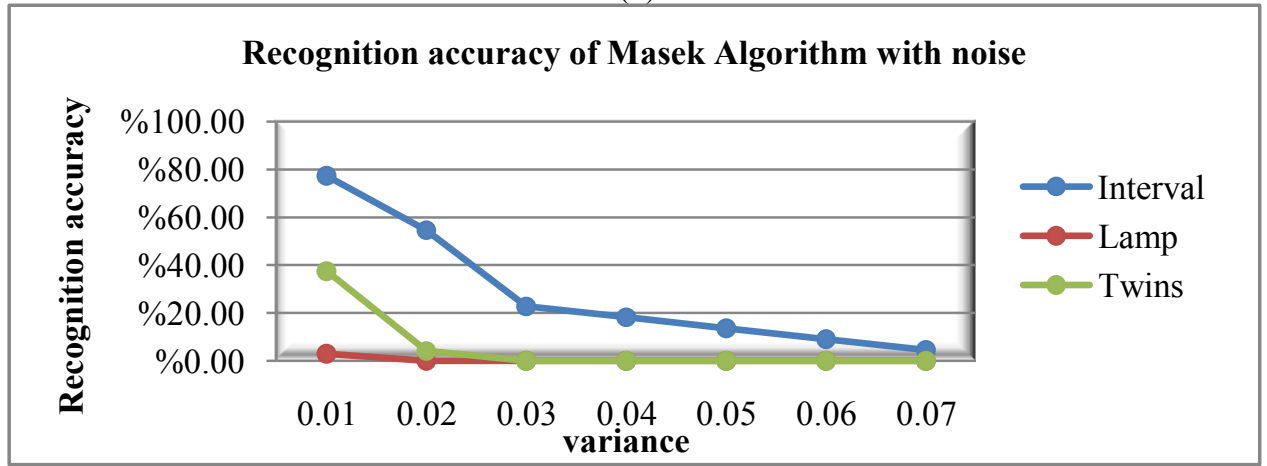

(d)

Figure 3 (a,b,c,d)Recognition accuracies for Alex, Vgg16 and Vgg19 in the presence of noise with variance value from 0.01 to 0.07 and compared to Masek result on CASIA-Iris V3 datasets.

\section{Conclusion}

This paper presented a comparative study of the performance of three deep learning models for iris recognition; Alex net, Vgg16 and Vgg19 in the presence of noise and compare them with a classical algorithm (Masek). The comparison confirms that the deep learning models are the optimum. Based on the interval database CHT accuracy was $68.18 \%$, but the accuracy of three deep learning models were $100 \%, 97.88 \%$ and $97.5 \%$,respectively.
Then after increasing the noise variance, the accuracy of CHT algorithm was reduced to $4.5 \%$ at variance of 0.07 , but these models accuracies are slightly reduced to $89.88 \%$, $91.54 \%$ and $93.61 \%$.

\section{References}

[1] A. K. Jain, A. Ross and S. Prabhakar, "An Introduction to Biometric Recognition", IEEE Transactions on Circuits and Systems for Video Technology 14(1), 4-20, 2004.

[2] J. L. Wayman, A. Jain, D. Maltoni, and D. Maio, "Biometric Systems, Technology, Design and Performance Evaluation", Springer 2005.

[3] M. Essam, M.Fikri, M. AbdElnaby, F. E. Abd El-Samie "An Efficient Iris Localization Algorithm", 29th National Radio Science Conference, Egypt (NRSC2012), pp. 285-292, 2012. 
[4] J. Daugman, "How Iris Recognition Works", IEEE Transaction on Circuits and Systems for Video Technology 14(1),21-30, 2004.

[5] R. Wildes, "Iris Recognition: An Emerging Biometric Technology", Proceedings of the IEEE 85(9),pp. 1348-1363, 1997.

[6] L. Masek, "Recognition of Human Iris Patterns for Biometric Identification", MSc. Thesis, the University of Western Australia, 2003.

[7] C. Li, C. Xu, C. Gui, and D. F. Martin, "Distance Regularized Level Set Evolution and Its Application to Image Segmentation", IEEE Transaction on Image Processing 19(12), 3243-4254, 2010.

[8] P. Verma, M. Dubey, S. Basu, and P. Verma, "Hough Transform Method for Iris Recognition-A Biometric Approach", International Journal of Engineering and Innovative Technology (IJEIT) 1(6),4348, 2012.

[9] N. Cherabit, F. Chelali, and A. Djeradi, "Circular Hough Transform for Iris localization", Science and Technology 2(5), 114-121, 2012.

[10] A. Gangwar and A. Joshi, "Deepirisnet: Deep iris representation with applications in iris recognition and cross-sensor iris recognition", 2016 IEEE International Conference on Image Processing (ICIP),pp. 2301-2305, 2016.

[11] S. Minaee, A. Abdolrashidiy, and Y. Wang, "An experimental study of deep convolutional features for iris recognition,” 2016 IEEE Signal Processing in Medicine and Biology Symposium (SPMB), pp. 1-6, 2016.

[12] A. S. Razavian, H. Azizpour, J. Sullivan, and S. Carlsson, "Cnn features off-the-shelf: An astounding baseline for recognition", 2014 IEEE Conference on Computer Vision and Pattern Recognition Workshops, pp. 512-519, 2014.

[13] J. Schmidhuber, "Deep learning in neural networks: An overview", Neural Networks 61, 85-117, 2015.

[14] Y. Bengio, A. Courville, and P. Vincent, "Representation learning: A review and new perspectives", IEEE Transactions on Pattern Analysis and Machine Intelligence 35(8), 1798-1828, 2013.

[15] A. Canziani, A. Paszke, and E. Culurciello, "An analysis of deep neural network models for practical applications", CoRR, vol. abs/1605.07678, 2016.

[16] A. Krizhevsky, I. Sutskever, and G. E. Hinton, "Imagenet classification with deep convolutional neural networks", Advances in neural information processing systems 25(2), 1097-1105, 2012.

[17] K. Simonyan and A. Zisserman, "Very deep convolutional networks for large-scale image recognition," CoRR, vol. abs/1409.1556, 2014.

[18] C. Szegedy, W. Liu, Y. Jia, P. Sermanet, S. Reed, D. Anguelov, D. Erhan, V. Vanhoucke, and A. Rabinovich, "Going deeper with convolutions", 2015 IEEE Conference on Computer Vision and Pattern Recognition (CVPR), pp. 1-9, 2015

[19] K. He, X. Zhang, S. Ren, and J. Sun, "Deep residual learning for image recognition", 2016 IEEE Conference on Computer Vision and Pattern Recognition (CVPR), pp. 770-778, 2016.

[20] G. Huang, Z. Liu, K. Q. Weinberger, and L. van der Maaten, "Densely connected convolutional networks", 2016 IEEE Conference on Computer Vision and Pattern Recognition (CVPR), pp. 4700-4708, 2017.

[21] N. Liu, M. Zhang, H. Li, Z. Sun, and T. Tan, "Deepiris: Learning pairwise filter bank for heterogeneous iris verification", Pattern Recognition Letters 82, 154-161, 2016.

[22] CASIA-IrisV3 database, available at: http://www.cbsr.ia.ac.cn/english/IrisDatabase.asp [Online, accessed April 2015].

[23] P. J. Phillips, W. T. Scruggs, A. J. O’Toole, P. J. Flynn, K. W. Bowyer, C. L. Schott, and M. Sharpe, "Frvt 2006 and ice 2006 largescale experimental results," IEEE Transactions on Pattern Analysis and Machine Intelligence 32(5), 831-846, 2010.

[24] J. Tapia and C. Aravena, "Gender Classification from NIR Iris Images Using Deep Learning”, In: Bhanu B., Kumar A. (eds) Deep Learning for Biometrics. Advances in Computer Vision and Pattern Recognition. Springer, Cham, pp. 219-239, 2017.

[25] N. Liu, H. Li, M. Zhang, J. Liu, Z. Sun, and T. Tan, “Accurate iris segmentation in non-cooperative environments using fully convolutional networks", 2016 International Conference on Biometrics (ICB),pp. 1-8, 2016.

[26] E. Jalilian and A. Uhl, "Iris Segmentation Using Fully Convolutional Encoder-Decoder Networks", In: Bhanu B., Kumar A. (eds) Deep Learning for Biometrics. Advances in Computer Vision and Pattern Recognition. Springer, Cham, 133-155, 2017.

[27] L. He, H. Li, F. Liu, N. Liu, Z. Sun, and Z. He, "Multi-patch convolution neural network for iris liveness detection", 2016 IEEE 8th International Conference on Biometrics Theory, Applications and Systems (BTAS), pp. 1-7, 2016.

[28] D. Menotti, G. Chiachia, A. Pinto, W. R. Schwartz, H. Pedrini, A. X. Falco, and A. Rocha, "Deep representations for iris, face, and fingerprint spoofing detection", IEEE Transactions on Information Forensics and Security 10(4), 864-879, 2015.

[29] M. D. Zeiler and R. Fergus, Visualizing and Understanding Convolutional Networks. Springer International Publishing, pp. 818833, 2014.

[30] O. Russakovsky, J. Deng, H. Su, J. Krause, S. Satheesh, S. Ma, Z. Huang, A. Karpathy, A. Khosla, M. Bernstein, A. C. Berg, and L. FeiFei, "Imagenet large scale visual recognition challenge", International Journal of Computer Vision 115(3), 211-252, 2015.

[31] B. Scholkopf and A. J. Smola, "Learning with Kernels: Support Vector Machines, Regularization, Optimization, and Beyond", MIT Press Cambridge, MA, USA, 2001. 